INPLASY

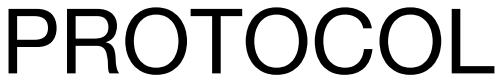

To cite: Wang et al.

Acupuncture for diabetic neuropathic pain: protocol for a systematic review and metaanalysis. Inplasy protocol 202090043. doi:

10.37766/inplasy2020.9.0043

Received: 10 September 2020

Published: 10 September 2020

Corresponding author:

Liqin Wang

wang_liqin0451@163.com

Author Affiliation:

First Affiliated Hospital, Heilongjiang University of Chinese Medicine

Support: SJGY20180469, HLJUCM2018RCD02.

Review Stage at time of this submission: Preliminary searches.

Conflicts of interest:

None.

\section{Acupuncture for diabetic neuropathic pain: protocol for a systematic review and meta-analysis}

Wang, L1; Gao, Z2; Niu, X33 Yuan, M4; Li, Y5; Wang, F6; Guo, C7; Ren, Z8. $^{8}$

Review question / Objective: There are several small sample size randomized controlled trials concerning acupuncture in treating diabetic neuropathic pain (DNP), however, to the best of our knowledge, there is no systematic review to summaries the results. Therefore, a comprehensive review of acupuncture in treating DNP is needed, and the evidence could provide more options for DNP management.

Condition being studied: DNP is one of the most common complications of diabetes mellitus, affecting approximately $30 \%-50 \%$ of diabetes patients. The main symptoms of DNP include spontaneous pain and irritation-induced pain. DNP is hard to treat. Complementary and alternative medicine interventions are being used to treat DNP.

INPLASY registration number: This protocol was registered with the International Platform of Registered Systematic Review and Meta-Analysis Protocols (INPLASY) on 10 September 2020 and was last updated on 10 September 2020 (registration number INPLASY202090043).

\section{INTRODUCTION}

Review question / Objective: There are several small sample size randomized controlled trials concerning acupuncture in treating diabetic neuropathic pain (DNP), however, to the best of our knowledge, there is no systematic review to summaries the results. Therefore, a comprehensive review of acupuncture in treating DNP is needed, and the evidence could provide more options for DNP management. 
Condition being studied: DNP is one of the most common complications of diabetes mellitus, affecting approximately $30 \%-50 \%$ of diabetes patients. The main symptoms of DNP include spontaneous pain and irritation-induced pain. DNP is hard to treat. Complementary and alternative medicine interventions are being used to treat DNP.

\section{METHODS}

Participant or population: Patients diagnosed with DNP will be included regardless of sex, age, race, education, and economic status.

Intervention: Acupuncture is defined as needle stimulation of acupoints, we will include studies using body acupuncture, scalp acupuncture, manual acupuncture, auricular acupuncture, electroacupuncture, fire needling et al.

Comparator: Control intervention will include any other treatments used to manage DNP other than acupuncture.

Study designs to be included: Randomized controlled trials (RCTs).

\section{Eligibility criteria: (1) Types of studies:}

RCTs; (2) Types of participants: Patients diagnosed with DNP; (3) Type of interventions: Acupuncture; and (4) Types of outcome measures: Primary outcomes: The primary outcome measure will be patient-reported pain intensity using validated scales. The secondary outcomes including the Toronto clinical scoring system (TCSS), Sensory Nerve Conduction Velocity ( SNCV), Motor Nerve Conduction Velocity(MNCV), and quality of life 36-Item Short Form Health Survey (SF-36).

Information sources: The reviewers will conduct a systematic literature search in the following electronic databases: PubMed, Embase, Web of Science, China National Knowledge Infrastructure (CNKI), Wan-Fang Database and Chinese Scientific Journal Database (VIP database).
Main outcome(s): The primary outcome measure will be patient-reported pain intensity using validated scales.

Quality assessment / Risk of bias analysis: Risk of bias of included study will be assessed using the Cochrane collaborative tool. We will evaluate the following aspects of the studies including: sequence generation, assignment sequence hiding, blindness of participants and staff, outcome evaluators, incomplete result data, selective result reporting, and other sources of bias. The risk of bias is evaluated at three levels, namely, low risk, high risk, and ambiguity. If the information is not clear enough, we will try to contact the author of the article for further information.

Strategy of data synthesis: We will use Review Manager Software (RevMan) V.5.3 for data synthesis, meta-analysis. Mean difference or standardized mean difference and $95 \%$ confidence intervals (Cls) will be used to calculate quantitative data, and dichotomous data will be exerted as risk ratio and $95 \%$ Cls. Statistical heterogeneity across studies will be done with 12 statistic. $12 \leq 50$ indicates homogeneity among studies, and a fixed-effects model will be employed for pooled analysis. $12>50 \%$ suggests obvious heterogeneity, and a random-effects model will be employed for synthesized analysis. When there is the homogeneity of the merged outcome results across sufficient studies, metaanalysis will be conducted.

Subgroup analysis: Subgroup analysis will be conducted based on the difference of interventions, controls, outcome measurements and et. al if necessary.

Sensibility analysis: Sensitivity analysis will be undertaken to check the stability of merged outcome results by excluding studies with high risk of bias if significant heterogeneity exists.

Country(ies) involved: China. 
Keywords: Diabetic neuropathic pain, Acupuncture, systematic review, metaanalysis.

Contributions of each author:

Author 1 - Liqin Wang.

Author 2 - Zhaohong Gao.

Author 3 - Xiangru Niu.

Author 4 - Meiqi Yuan.

Author 5 - Yan Li.

Author 6 - Fei Wang.

Author 7 - Chuang Guo.

Author 8 - Zhen Ren. 